


\section{Lehrbuch der Chemie}

Von

Dr. phil. Max Trautz

Professor für physikalische Chemie und Elektrochemie an der Universität Heidelberg

Gesamtumfang etwa roo Bogen in 3 Bänden

\section{Erster Band : \\ S $t$ o f $f$ e}

Mit zahlreichen Abbildungen im Texte und auf Tafeln und mit Tabellen

Groß-Oktav. XXVIII, 534 Seiten. 1922. Grundzahl I6

\section{Zweiter Band: \\ Z u s t ä n d e}

Mit zahlreichen Abbildungen im Text und auf Tafelu und nit Tabellen

Grof-Oktav. XXXIV, 634 Seiten

Erscheint Ende Januar 1923

\section{Dritter Band : \\ U m w a n d 1 ug e n}

Im Druck

Verkaufspreis: Grundzahl $\times$ Schlüsselzahl (am 17.Januar 1923 = 700)

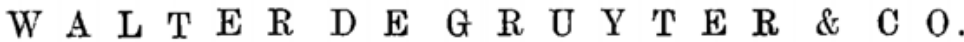
vORMALS G. J. GÖSCHEN'SCHE VERLAGSHANDLUNG / J. GUTTEN-

TAG, VERLAGSBUCHHANDLUNG / GEORG REIMER

KARL J, TRÜBNER / VEIT \& COMP.

B ERLIN W. 10 UND LEIPZIG 


\section{Praktische Einfuihrung in die allgemeine Chemie}

Anleitung zu physikalisch-chemischem Praktikum und selbständiger Arbeit. Von Prof. Dr. Max Trautz. Mit $187 \mathrm{Ab}-$ bildungen.

Grundzahl Io

$\mathrm{Zu}$ diesem Werke werden besonders geliefert:

Versuchs-Berichte zum Selbsteintragen Grundzahl I

\section{Die Praxis des organischen Chemikers}

Von Prof. Dr. Ludwig Gattermann. I7. Aufl. Mit $95 \mathrm{Ab}$ bildungen und 2 Tabellen. GroB-Oktav. XII, 368 Seiten. 1922 .

Grundzahl (nur gebunden) 8

\section{Praktikum des anorganischen Chemikers}

Einführung in die anorganische Chemie auf experimenteller Grundlage. Von Prof. Dr. Emil Knoevenagel. 3. Auflage. Mit zahlreichen Figuren, 4 Tabellen u. 9 Tafeln. GroB-Oktav. XXVIII, 386 Seiten. I920. Grundzahl (nur gebunden) I 2,7

\section{Anleitung zur Darstellung chemischer anorga- nischer Präparate}

Für Chemiker und Pharmazeuten. Von Prof. Dr. Reinhart Blochmann. Mit zahlreichen Figuren. 3. unverănderte Auflage. Oktav. X, I76 Seiten. I921.

Grundzahl (nur gebunden) 5

\section{Kurzes chemisches Praktikum für Mediziner und Landwirte}

Von Prof. Dr. Fritz Arndt. 5. und 6. Auflage. GroBOktav. VIII, 96 Seiten. I921. Grundzahl 2,8

Verkaufspreis: Grundzahl $\times$ Schlusselzahl (am 17. Januar $1923=700$ )

W A L T VORMALS G. J. GOSCHEN'SCEE YERLAGSHANDLUNG / J. GUTTEN-

TAG, VERLAGSBUCHHANDLUNG / GEORG REIMER

KARL J. TRÜBNER / VEIT \& COMP.

BERLIN W. 10 UND LEIPZIG 


\title{
Meyer-Jacobson
}

\section{Lehrbuch der organischen Chemie.}

\author{
In zwed Panden.
}

Erster Band: Allgemeiner Teil - Verbindungen der Fettreihe

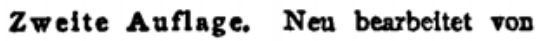

P. Jacobson und R. Stelzner

GroB-Oktav. Zwei Teile

Erster Tsil: Allgemeiner Tell. - Die allphatischen Koblen. wasserstoffe und ihre einwertigen Abkommlinge. Mit Figuren im Text. (XVI n. $1060 \mathrm{~S}$.)

Grondzahl 26.?

Zwoiter Teil: Die mehrwertigen Abkommlinge der alipha. tischen Kohlenwasserstoffe. - Cyanverbindungen nud Kohlensărederivate. Mit Figuren im Text und ciner beigehefteten Tabelle. (XXIV u. $1522 \mathrm{~S}$.) Grundrabl 38.8

\section{Zweiter Band: Cyclische Verbindungen - Naturstoffe}

$$
\text { Erste nnd zweite Anflage }
$$

Grob-Oktav. Drel Telle

Erster Teil: Elnkernige 1socyclische Verblndungen. Die Gruppe der hydroaromatischen Verbindungen ist in Gemeinschant mit P. Jacobson bearbeitet von Carl Harries. (XX u. ro76 S.)

Neudrack Mraz 1923

Zweiter Teil: Mehrkernige Benzolderivate. In Gemeinschaft mit P. Jacobson bearbeitet von Arnold ReiBert. (XIV u. $664 \mathrm{~S}$.)

Neudruck Mărz 1923

Dritter Teil: Heterocyclische Verbindungen. Bearbettet von $P$. Jacobson. (XXI घ. $1634 \mathrm{~S}$.) Neudrucls Mărz I923

Die Lioforungen von Tell IV des zwelten Bandes befinden sich in Vorbereitung und werden die heterocyclischen Verbindungen und die Naturstoffe unbekannter Konstitution enthalten.

Meyer-Jacobsons Lehrbuch ist neben dem andere wissenschaflicho Ziele verfolgenden Beilsteinschen Handbuch das klassischo Werk dor modernen organischen Chemio.

Die Lî́erztur wird bis is die neueste Zeit is größter Vollständigkeit gegeben.

Verkaufspreis: Grundzahl $\times$ Schlüsselzahl (am 17. Januar $1923=700)$

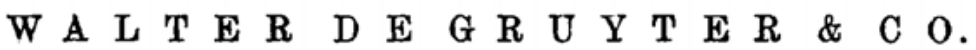
VORMALS G. J. GÖSCHEN' SCHE VERLAGSHANDLUNG / J. GUTTEN-

TAG, VERLAGSBUCHHANDLUNG I GEORG REIMER KARL J. TRUBNER / VEIT \& COMP.

BERLIN W. 10 UN D LEIPZIG 


\title{
LOGARITHMISCHE RECHENTAFELN
} FÜR CHEMIKER,

\section{PHARMAZEUTEN, MEDIZINER UND PHYSIKER.}

\author{
Begründet von \\ Professor Dr. F. W. Küster $\uparrow$.
}

Für den Gebrauch im Unterrichtslaboratorium und in der Praxis berechnet und mit Erläuterungen versehen.

Nach dem gegenwärtigen Stande der Forschung bearbeitet von

\section{Dr. A. Thiel,}

o. o. Professor der physikalischen Chemie,

Direktor des Physikalisch-chemischen Instituts der Universität Marburg.

27.-29., verbesserte und vermehrte Auflage.

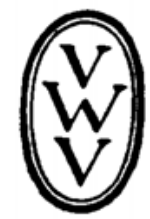

BERLIN und LEIPZIG 1923

W A L T E R D E G R U Y T E R \& C O. vormals G. J. Göschen'sche Verlagshandlung - J. Guttentag, Verlagsbuchhandlung - Georg Reimer -

Karl J. Trübner - Veit \& Comp. 


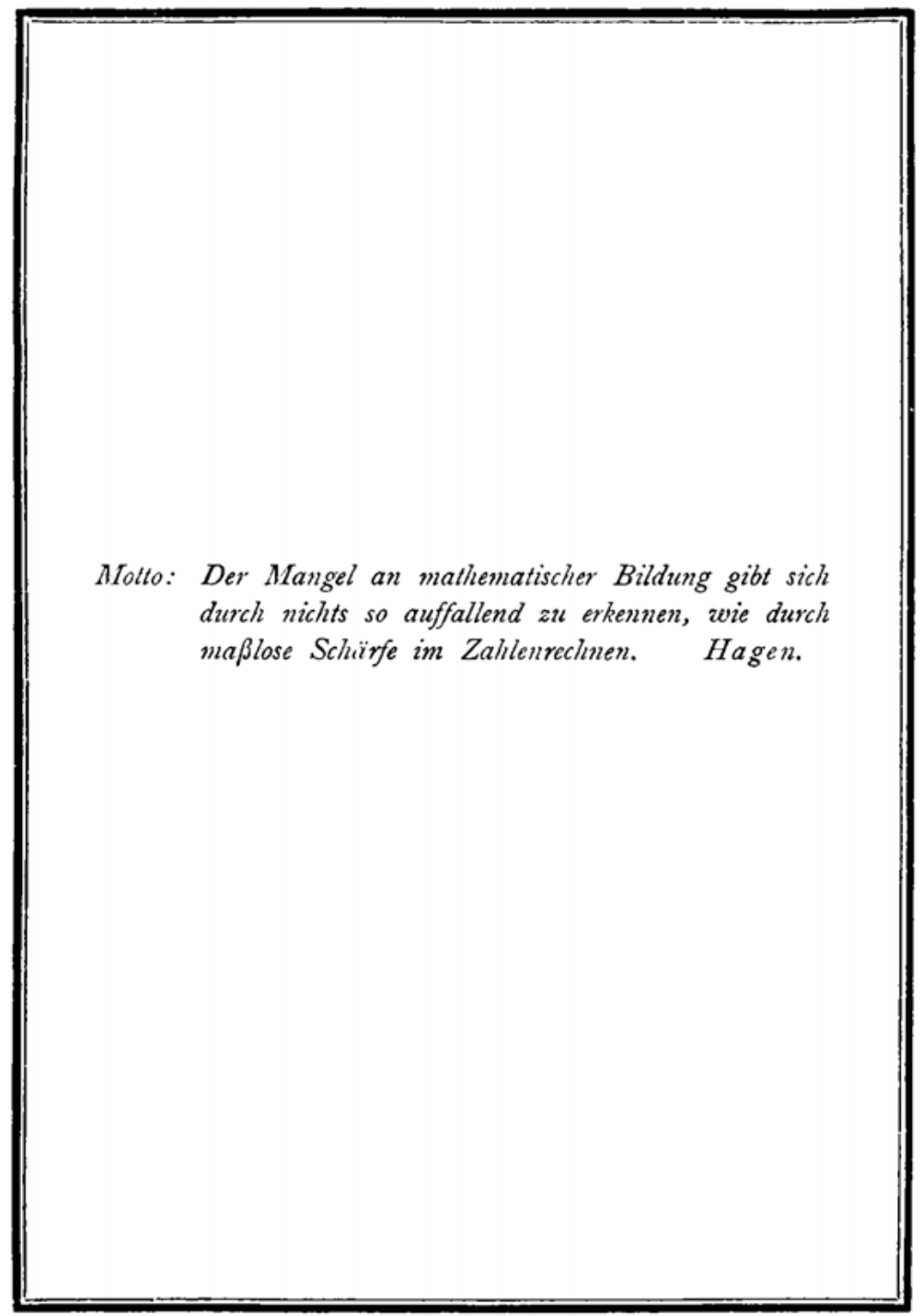

Druck von Metzger \& Wittig in Leipzig. 